\title{
ON FOURIER TRANSFORMS OF FUNCTIONS OF THE R. NEVANLINNA CLASS IN THE HALF-PLANE
}

\author{
F. A. SHAMOYAN
}

Abstract. Let $f$ be a function holomorphic in the upper half-plane and belonging to the Nevanlinna class $N\left(\mathbb{C}_{+}\right)$. Assume that

$$
\limsup _{y \rightarrow+\infty} \frac{\ln |f(i y)|}{y} \leq 0
$$

and that the boundary values of $f$ on the real axis lie in $L^{1}(\mathbb{R})$. It is shown that if $|\widehat{f}(x)| \leq \frac{1}{\lambda(|x|)}, x \in \mathbb{R}_{-}$, where $\widehat{f}$ is the Fourier transform of $f$ and $\lambda$ is a logarithmically convex positive function on $\mathbb{R}_{+}$, then the condition $\int_{1}^{+\infty} \frac{\ln \lambda(x)}{x^{3 / 2}} d x=+\infty$ implies that $\widehat{f}(x)=0$ for all $x \in \mathbb{R}_{-}$. Conversely, if one of the conditions listed above fails, then there exists $f \in N\left(\mathbb{C}_{+}\right) \cap L^{1}(\mathbb{R})$ with $\widehat{f}(x) \neq 0, x \in \mathbb{R}_{-}$.

We denote by $\mathbb{C}_{+}=\{z \in \mathbb{C}: \operatorname{Im} z>0\}$ the upper half-plane, by $H\left(\mathbb{C}_{+}\right)$the set of all functions holomorphic in $\mathbb{C}_{+}$, and by $H^{p}\left(\mathbb{C}_{+}\right)=H^{p}(0<p \leq+\infty)$ the Hardy class on $\mathbb{C}_{+}$.

It is easy to observe that if $f \in H\left(\mathbb{C}_{+}\right),\left.f\right|_{\mathbb{R}} \in L^{1}(\mathbb{R})$, and there exists a nonnegative harmonic function $U$ in $\mathbb{C}_{+}$such that

$$
|f(z)|^{p_{0}} \leq U(z), \quad z \in \mathbb{C}_{+},
$$

for some $p_{0} \in(0,+\infty)$, then, by the V. I. Smirnov theorem (see [1, 2]), we have $f \in$ $H^{1}\left(\mathbb{C}_{+}\right)$, and therefore, the Fourier transform

$$
\widehat{f}(x)=\frac{1}{\sqrt{2 \pi}} \int_{-\infty}^{+\infty} f(t) e^{-i t x} d t, \quad x \in \mathbb{R},
$$

vanishes on $\mathbb{R}_{-}=(-\infty, 0)$.

The following question arises naturally: is a similar statement true if we replace $|f|^{p_{0}}$ by $\ln ^{+}|f|$ in (1)? Simple examples show that there exists $f \in H\left(\mathbb{C}_{+}\right)$and a nonnegative harmonic function $U$ such that

$$
\ln ^{+}|f(z)| \leq U(z), \quad z \in \mathbb{C}_{+},
$$

$\left.f\right|_{\mathbb{R}} \in L^{1}(\mathbb{R})$, and $\widehat{f}(x) \neq 0, x \in \mathbb{R}_{-}$. We note that the class of functions satisfying (2) coincides with the Nevanlinna class of functions of bounded characteristic (see [2]); we denote this class by $N\left(\mathbb{C}_{+}\right)$.

In this paper, we establish that, under the same conditions, if $\widehat{f}(x) \rightarrow 0$ sufficiently fast as $x \rightarrow-\infty$, then $\widehat{f}(x)=0$ for all $x \in \mathbb{R}_{-}$. Also, we find a complete description of the monotone increasing functions for which the condition $|\widehat{f}(x)| \leq \frac{1}{\lambda(|x|)}, x \in \mathbb{R}_{-}$, implies that $\widehat{f}(x)=0, x \in \mathbb{R}_{-}$.

The main results of this paper were announced in $[3$.

2000 Mathematics Subject Classification. Primary 30D50.

Key words and phrases. Function of bounded characteristic, Fourier transform. 


\section{$\S 1$. Formulation of the main RESUlts AND PROOF OF AUXiLiary STATEMENTS}

Let $\lambda$ be a monotone increasing, continuous, and positive function on $\mathbb{R}_{+}$; we assume that

$$
\lim _{x \rightarrow+\infty} \frac{x^{n}}{\lambda(x)}=0, \quad n=1,2, \ldots .
$$

We call such a function a rapidly growing weight and denote

$$
m_{n}=\sup _{x>0} \frac{x^{n}}{\lambda(x)}, \quad T(r)=\sup _{n \geq 1} \frac{r^{n}}{m_{n}}, \quad r \geq 0 .
$$

Theorem 1. Let $\lambda(x)$ be a rapidly growing weight on $\mathbb{R}_{+}$. Suppose that $f \in N\left(\mathbb{C}_{+}\right) \cap$ $L^{1}(\mathbb{R})$ satisfies

$$
\int_{1}^{+\infty} \frac{\ln T(r)}{r^{3 / 2}} d r=+\infty
$$

and

$$
|\widehat{f}(x)| \leq \frac{1}{\lambda(-x)}, \quad x \in \mathbb{R}_{-} .
$$

Under these conditions, if

$$
\limsup _{y \rightarrow+\infty} \frac{\ln |f(i y)|}{y} \leq 0,
$$

then $\widehat{f}(x)=0$ for all $x \in \mathbb{R}_{-}$, and thus, $f \in H^{1}\left(\mathbb{C}_{+}\right)$.

Conversely, if $\lambda$ is a logarithmically convex and rapidly growing weight such that the integral (4) converges, then there exists $f \in N\left(\mathbb{C}_{+}\right) \cap L^{1}(\mathbb{R})$ such that estimate (5) is fulfilled, but $\widehat{f}(x) \neq 0, x \in \mathbb{R}_{-}$.

Combining Theorem 1 with estimates for the Carleman-Ostrowski function in the case of a logarithmically convex weight, we obtain the following statement.

Theorem 2. Let $\lambda$ be a logarithmically convex weight. The following statements are equivalent:

1) for every $f \in N\left(\mathbb{C}_{+}\right) \cap L^{1}(\mathbb{R})$ satisfying (5) and (6) , we have

$$
\begin{gathered}
\widehat{f}(x)=0, \quad x \in \mathbb{R}_{-} ; \\
\int_{1}^{+\infty} \frac{\ln \lambda(x)}{x^{3 / 2}} d x=+\infty .
\end{gathered}
$$

Remark 1. In Theorem 1, condition (6) cannot be lifted. As a simple example in which all conditions of Theorem 1 except (6) are fulfilled, we mention the functions

$$
f_{a}(z)=\frac{1}{e^{i a z}(i N+z)^{2}}, \quad a>0, \quad N>0, \quad z \in \mathbb{C}_{+} .
$$

Obviously, supp $\widehat{f}_{a} \cap \mathbb{R}_{-} \neq \varnothing$.

Remark 2. We also note that the condition $f \in N\left(\mathbb{C}_{+}\right)$is necessary in a sense: in [5] it was established that for any measurable function $\psi$ finite almost everywhere on $\mathbb{R}$ and any function $h(x)>0$ with $\lim _{x \rightarrow 0} h(x)=+\infty$, there exists $f \in H\left(\mathbb{C}_{+}\right)$such that $|f(x+i y)| \leq h(y)$ and

$$
\lim _{y \rightarrow 0} f(x+i y)=\psi(x)
$$

almost everywhere. 
Remark 3. The rate of decay for the Fourier coefficients of functions of bounded characteristic was studied in the author's paper [6].

The example (7) shows that there exist functions $f \in N\left(\mathbb{C}_{+}\right) \cap L^{1}(\mathbb{R}) \cap C^{\infty}(\mathbb{R})$ such that $M_{k}=\sup _{x \in \mathbb{R}}\left|f^{(k)}(x)\right|<+\infty, \lim _{|x| \rightarrow+\infty} f^{(k)}(x)=0, k=0,1,2, \ldots$, and

$$
\sup _{z \in \mathbb{C}_{+}}|f(z)|=+\infty \text {. }
$$

As usual, $C^{\infty}\left(M_{n}\right)$ denotes the Carleman class on $\mathbb{R}$ (see [4]), i.e.,

$$
C^{\infty}\left(M_{n}\right)=\left\{f \in C^{\infty}(\mathbb{R}):\left|f^{(k)}(x)\right| \leq A_{f}^{k} M_{k}, x \in \mathbb{R}, k=0,1, \ldots\right\} .
$$

It is natural to ask under what restrictions on the monotone increasing sequence $\left\{M_{k}\right\}_{k=1}^{\infty}$, $M_{k}>0, k=1,2, \ldots$, the condition $f \in N\left(\mathbb{C}_{+}\right) \cap L^{1}(\mathbb{R}) \cap C^{\infty}\left(M_{n}\right)$ implies

$$
\left|f^{(k)}(z)\right| \leq A_{f}^{k} M_{k}, \quad z \in \mathbb{C}_{+} .
$$

Theorem 3. Suppose $f \in N\left(\mathbb{C}_{+}\right) \cap L^{1}(\mathbb{R}) \cap C^{\infty}\left(M_{n}\right)$ and

$$
\limsup _{y \rightarrow+\infty} \frac{\ln |f(i y)|}{y} \leq 0 .
$$

If

$$
\int_{1}^{+\infty} \frac{\ln T(r)}{r^{3 / 2}} d r=+\infty
$$

where $T(r)=\sup _{k \geq 1} \frac{r^{k}}{M_{k}}, r \in \mathbb{R}_{+}$, then estimate (8) is valid.

Conversely, if at least one of the conditions (9), (10) fails, then there exists $f \in$ $N\left(\mathbb{C}_{+}\right) \cap L^{1}(\mathbb{R}) \cap C^{\infty}\left(M_{n}\right)$ such that $\sup _{z \in \mathbb{C}_{+}}|f(z)|=+\infty$.

The proofs of Theorems 1-3 are based on several auxiliary statements.

For $m>-1$ we put

$$
A^{1}(m)=\left\{f \in H\left(\mathbb{C}_{+}\right):\|f\|_{A^{1}(m)}=\int_{\mathbb{C}_{+}}(\operatorname{Im} z)^{m}|f(z)| d m_{2}(z)<\infty\right\} .
$$

Here $m_{2}$ denotes the Lebesgue measure on $\mathbb{R}^{2}$.

Lemma 1. Any $f \in A^{1}(m)$ can be represented in the form

$$
f(z)=c_{m} \int_{\mathbb{C}_{+}} \frac{(\operatorname{Im} \zeta)^{m} f(\zeta) d m_{2}(\zeta)}{(\bar{\zeta}-z)^{m+2}} \text {, where } c_{m}=-\frac{(m+1) 2^{m-1}}{\pi} e^{\left(-i(m-1) \frac{\pi}{2}\right)} .
$$

Proof. Formula (11) follows directly from the Cauchy-Green formula. It suffices to check (11) for a set dense in $A^{1}(m)$.

Let, for instance, $F \in W_{1}^{1}\left(\mathbb{C}_{+}\right) \cap C_{0}\left(\mathbb{C}_{+} \cup \mathbb{R}\right)$, where $W_{1}^{1}\left(\mathbb{C}_{+}\right)$is the Sobolev class on $\mathbb{C}_{+}$, and $C_{0}\left(\mathbb{C}_{+} \cup \mathbb{R}\right)$ is the class of functions that are continuous in $\mathbb{C}_{+} \cup \mathbb{R}$ and tend to zero sufficiently fast as $z \rightarrow \infty$. The Cauchy-Green formula (see, e.g., [7, Chapter 1]) yields

$$
F(w)=\frac{1}{2 \pi i} \int_{\mathbb{C}_{+}} \frac{\partial F(\zeta)}{\partial \bar{\zeta}} \frac{d m_{2}(\zeta)}{\zeta-w}+\frac{1}{2 \pi i} \int_{\mathbb{R}} \frac{F(x)}{x-w} d x .
$$

Next, assume that $f \in H\left(\mathbb{C}_{+}\right) \cap W_{1}^{1}\left(\mathbb{C}_{+}\right) \cap C_{0}\left(\mathbb{C}_{+} \cup \mathbb{R}\right)$. Put

$$
F_{z}(w)=f(w)\left(\frac{w-\bar{w}}{\bar{w}-z}\right)^{m+1}, \quad \text { where } w, z \in \mathbb{C}_{+} .
$$

Applying (12) to $F_{z}(w)$ and putting $w=z$, we arrive at formula (11). 
In the general case, for an arbitrary $f \in A^{1}(m)$, we set

$$
f_{N}(z)=f(z) \frac{N^{p}}{(i N+z)^{p}}, \quad z \in \mathbb{C}_{+},
$$

where $N$ and $p$ are sufficiently large positive integers. Writing the representation (11) for $f_{N}$ and letting $N \rightarrow+\infty$, we complete the proof of the lemma.

Remark 4. Note that in 8 , the representation (11) was established in another way.

To formulate the next statement, we denote

$$
C_{A}^{\infty}\left(\mathbb{C}_{-}\right)=\left\{f \in H\left(\mathbb{C}_{-}\right) \cap C^{\infty}\left(\mathbb{C}_{-} \cup \mathbb{R}\right): \sup _{z \in \mathbb{C}_{-}}\left|f^{(n)}(z)\right|<\infty, \quad n=0,1,2, \ldots\right\},
$$

where $\mathbb{C}_{-}=\{z \in \mathbb{C}: \operatorname{Im} z<0\}$.

Lemma 2. If $f \in C_{A}^{\infty}\left(\mathbb{C}_{-}\right)$, then the limit

$$
\Phi(g)=\lim _{y \rightarrow 0+} \int_{-\infty}^{+\infty} f(x-i y) g(x+i y) d x
$$

exists for any $m \in \mathbb{Z}_{+}$and any $g \in A^{1}(m)$.

We have $\Phi \in\left(A^{1}(m)\right)^{*}$ and $\|\Phi\| \leq C_{1}(m) \sup _{z \in \mathbb{C}_{-}}\left|f^{(m+1)}(z)\right|$.

Proof. Fix $y>0$. By Lemma 1,

$$
\begin{gathered}
\int_{-\infty}^{+\infty} f(x-i y) g(x+i y) d x=c_{m} \int_{-\infty}^{+\infty} f(x-i y)\left(\int_{\mathbb{C}_{+}} \frac{g(\xi+i \eta) \eta^{m}}{(\xi-i \eta-x-i y)^{m+2}} d \xi d \eta\right) d x \\
=c_{m} \int_{\mathbb{C}_{+}} g(\xi+i \eta) \eta^{m}\left(\int_{-\infty}^{+\infty} \frac{f(x-i y)}{(\xi-i \eta-x-i y)^{m+2}} d x\right) d \xi d \eta
\end{gathered}
$$

where $c_{m}$ is the constant occurring in (11). We evaluate the inner integral. We have

$$
\int_{-\infty}^{+\infty} \frac{f(x-i y)}{(\xi-i \eta-x-i y)^{m+2}} d x=(-1)^{m} \int_{-\infty}^{+\infty} \frac{f_{-y}(x)}{(x-(\xi-i(\eta+y)))^{m+2}} d x
$$

where $f_{-y}(x)=f(x-i y), y>0$. Clearly, under the assumptions of the lemma the integral on the right is equal to

$$
\frac{(-1)^{m} 2 \pi i f^{(m+1)}(\xi-i(\eta+2 y))}{(m+1) !} .
$$

Therefore,

$$
\int_{-\infty}^{+\infty} f(x-i y) g(x+i y) d x=\frac{(-1)^{m} c_{m} 2 \pi i}{(m+1) !} \int_{\mathbb{C}_{+}}(\operatorname{Im} \zeta)^{m} g(\zeta) f^{(m+1)}(\bar{\zeta}-2 i y) d m_{2}(\zeta) .
$$

This identity implies that

$$
\begin{aligned}
\Phi(g) & =\lim _{y \rightarrow 0+} \int_{-\infty}^{+\infty} f(x-i y) g(x+i y) d x \\
& =\frac{(-1)^{m} c_{m} 2 \pi i}{(m+1) !} \int_{\mathbb{C}_{+}} g(\zeta) f^{(m+1)}(\bar{\zeta})(\operatorname{Im} \zeta)^{m} d m_{2}(\zeta) .
\end{aligned}
$$

Thus,

$$
|\Phi(g)| \leq C_{1}(m)\|g\|_{A^{1}(m)} \sup _{z \in \mathbb{C}_{-}}\left|f^{(m+1)}(z)\right|
$$


We shall need the following formula for the derivatives of composition (see 9, Chapter 1]):

$$
(g(f(z)))^{(n)}=\sum \frac{n !}{m_{1} ! \cdots m_{q} !} g^{(p)}(f(z))\left(\frac{f^{(1)}(z)}{1 !}\right)^{m_{1}} \cdots\left(\frac{f^{(q)}(z)}{q !}\right)^{m_{q}} .
$$

The sum is over all positive integers such that $m_{1}+2 m_{2}+\cdots+q m_{q}=n$, and $p=$ $m_{1}+m_{2}+\cdots+m_{q}$.

Lemma 3. If $I_{n}=\sum_{\substack{m_{1}+2 m_{2}+\\ \cdots+q m_{q}=n}} \frac{n !}{m_{1} ! \cdots m_{q} !}$, then $I_{n} \leq n ! 2^{n} e$.

Proof. For $g(z)=e^{z}$ and $f(z)=-\frac{1}{1+z}$, formula (13) yields

$$
\left(e^{f(z)}\right)^{(n)}=\sum_{\substack{m_{1}+2 m_{2}+\\ \cdots+q m_{q}=n}} \frac{n !}{m_{1} ! \cdots m_{q} !} e^{f(z)}\left(\frac{f^{(1)}(z)}{1 !}\right)^{m_{1}} \ldots\left(\frac{f^{(q)}(z)}{q !}\right)^{m_{q}} .
$$

Since $f^{(j)}(z)=\frac{(-1)^{j+1} j !}{(1+z)^{j+1}}$ and $\frac{f^{(j)}(-2)}{j !}=\frac{(-1)^{j+1}}{(-1)^{j+1}}=1$, we obtain

$$
\left(e^{f(z)}\right)_{z=-2}^{(n)}=e \sum_{\substack{m_{1}+2 m_{2}+\\ \cdots+q m_{q}=n}} \frac{n !}{m_{1} ! \cdots m_{q} !}=e I_{n} .
$$

On the other hand, by the Cauchy formula,

$$
\left(e^{-\frac{1}{1+z}}\right)^{(n)}=\frac{n !}{2 \pi i} \int_{|\zeta+2|=\frac{1}{2}} \frac{e^{f(\zeta)}}{(\zeta-z)^{n+1}} d \zeta
$$

Therefore, (15) implies the estimate

$$
\begin{aligned}
e I_{n} & =\left|\frac{n !}{2 \pi i} \int_{|\zeta+2|=\frac{1}{2}} \frac{e^{f(\zeta)}}{(\zeta+2)^{n+1}} d \zeta\right| \leq \frac{n !}{2 \pi} 2^{n+1} \int_{|\zeta+2|=\frac{1}{2}}\left|e^{f(\zeta)}\right||d \zeta| \\
& \leq \frac{n ! 2^{n}}{\pi} \max _{|\zeta+2|=\frac{1}{2}}\left|e^{f(\zeta)}\right| \pi=n ! 2^{n} \max _{|\zeta+2|=\frac{1}{2}}\left|e^{-\frac{1}{1+\zeta}}\right| \leq n ! 2^{n} e^{2},
\end{aligned}
$$

completing the proof.

We put $S(z)=\exp \left(\frac{i}{\pi} \int_{-\infty}^{+\infty} \frac{1+t z}{t-z} \frac{d \nu(t)}{1+t^{2}}\right)$, where $\nu$ is a singular nonnegative measure on $\mathbb{R}$ with $\int_{-\infty}^{+\infty} \frac{d \nu(t)}{1+t^{2}}<+\infty$.

Lemma 4. Let $e_{\lambda}(z)=e^{i \lambda z}$ for $z \in \mathbb{C}_{+}, \lambda \in \mathbb{R}$. Then

$$
\left(e_{\lambda}(z) S^{\delta}(z)\right)^{(n)}=e_{\lambda}(z) S^{\delta}(z) \psi_{\delta, n, \lambda}(z), \quad z \in \mathbb{C}_{+} .
$$

Here $\psi_{\delta, n, \lambda} \in H\left(\mathbb{C}_{+}\right)$and

$$
\left|\psi_{\delta, n, \lambda}(z)\right| \leq c(n, \lambda) \begin{cases}\sum_{p=1}^{n}(U(z) \delta y)^{p}+1 & \text { if } \quad y \geq 1, \\ \frac{1}{y^{n}} \sum_{p=1}^{n}(y U(z) \delta)^{p} & \text { if } \quad 0<y<1,\end{cases}
$$

where $U(z)=\int_{-\infty}^{+\infty} \frac{d \nu(t)}{|t-z|^{2}}, z \in \mathbb{C}_{+}$.

Proof. Applying the Leibniz formula, we obtain

$$
\left(e^{i \lambda z} S_{\delta}(z)\right)^{(n)}=\sum_{k=0}^{n}\left(\begin{array}{l}
n \\
k
\end{array}\right) S_{\delta}^{(k)}(z)\left(e^{i \lambda z}\right)^{(n-k)}=\sum_{k=0}^{n}\left(\begin{array}{l}
n \\
k
\end{array}\right)(i \lambda)^{n-k} e^{i \lambda z} S_{\delta}^{(k)}(z), \quad z \in \mathbb{C}_{+},
$$

where for convenience we have denoted $S_{\delta}(z) \stackrel{\text { def }}{=} S^{\delta}(z), z \in \mathbb{C}_{+} \cup \mathbb{R}$. 
Now we apply (14) to the function

$$
f(z)=\frac{i \delta}{\pi} \int_{-\infty}^{+\infty} \frac{1+t z}{t-z} \frac{d \nu(t)}{1+t^{2}}
$$

and use the identity

$$
f^{(k)}(z)=\frac{i k !}{\pi} \int_{-\infty}^{+\infty} \frac{d \nu(t)}{(t-z)^{k+1}}, \quad z \in \mathbb{C}_{+}, \quad k \in \mathbb{N} .
$$

This yields

$$
\begin{aligned}
S_{\delta}^{(k)}(z)=\sum_{m_{1}+\cdots+q m_{q}=k} & \frac{k !}{m_{1} ! \cdots m_{q} !} S_{\delta}(z)\left(\frac{i \delta}{\pi}\right)^{m_{1}+m_{2}+\cdots+m_{q}} \\
& \times\left(\int_{-\infty}^{+\infty} \frac{d \nu(t)}{(t-z)^{2}}\right)^{m_{1}} \cdots\left(\int_{-\infty}^{+\infty} \frac{d \nu(t)}{(t-z)^{q+1}}\right)^{m_{q}} .
\end{aligned}
$$

Put

$$
\begin{aligned}
& \psi_{\delta, n, \lambda}(z)=\sum_{k=1}^{n}\left(\begin{array}{l}
n \\
k
\end{array}\right)(i \lambda)^{n-k}\left(\sum_{\substack{m_{1}+2 m_{2}+\\
\cdots+q m_{q}=k}} \frac{k !}{m_{1} ! \cdots m_{q} !}\left(\frac{i \delta}{\pi}\right)^{m_{1}+\cdots+m_{q}}\right. \\
&\left.\times\left(\int_{-\infty}^{+\infty} \frac{d \nu(t)}{(t-z)^{2}}\right)^{m_{1}} \cdots\left(\int_{-\infty}^{+\infty} \frac{d \nu(t)}{(t-z)^{q+1}}\right)^{m_{q}}\right)+(i \lambda)^{n}, \\
& \lambda \in \mathbb{R}, \quad z \in \mathbb{C}_{+} .
\end{aligned}
$$

Then, by (16), we have

$$
\left(e^{i \lambda z} S_{\delta}(z)\right)^{(n)}=e^{i \lambda z} S_{\delta}(z) \psi_{\delta, n, \lambda}(z) .
$$

Obviously, $\psi_{\delta, n, \lambda} \in H\left(\mathbb{C}_{+}\right)$. To estimate this function, we use (17):

$$
\begin{aligned}
\left|\psi_{\delta, n, \lambda}(z)\right| \leq \sum_{k=1}^{n}\left(\begin{array}{l}
n \\
k
\end{array}\right)|\lambda|^{n-k} & \left(\sum_{\substack{m_{1}+2 m_{2}+\\
\cdots+q m_{q}=k}} \frac{k !}{m_{1} ! \cdots m_{q} !}\left(\frac{\delta}{\pi}\right)^{m_{1}+\cdots+m_{q}}\right. \\
& \left.\times\left(\int_{-\infty}^{+\infty} \frac{d \nu(t)}{|t-z|^{2}}\right)^{m_{1}+m_{2}+\cdots+m_{q}} \frac{1}{y^{m_{2}+\cdots+(q-1) m_{q}}}\right)+|\lambda|^{n} .
\end{aligned}
$$

We have used the inequality $|t-z| \geq y, z \in \mathbb{C}_{+}, t \in \mathbb{R}$. Since $m_{2}+2 m_{3}+\cdots+(q-1) m_{q}=$ $m_{1}+2 m_{2}+\cdots+q m_{q}-m_{1}-m_{2}-\cdots-m_{q}=k-p, p=m_{1}+m_{2}+\cdots+m_{q}$, estimate (18) leads to the bound

$$
\left|\psi_{\delta, n, \lambda}(z)\right| \leq C(n, \lambda)\left\{1+\sum_{k=1}^{n}\left(\sum_{\substack{m_{1}+\cdots+q m_{q}=k \\ p=m_{1}+\cdots+m_{q}}} \frac{k !}{m_{1} ! \cdots m_{q} !} \frac{\delta^{p}(U(z))^{p} y^{p}}{y^{k}}\right)\right\} .
$$

It remains to apply Lemma 3.

Lemma 5. Let $S(z)=\exp \left(\frac{i}{\pi} \int_{-\infty}^{+\infty} \frac{1+t z}{t-z} \frac{d \nu(t)}{1+t^{2}}\right)$ be a singular inner function on $\mathbb{C}_{+}$, and let $\delta>1$. Next, put

$$
\varphi_{N, n, \lambda, \delta}(z)=\frac{\left(S^{\delta}(z) e^{i \lambda z}\right)^{(n)} N^{2}}{(i N+z)^{2}}, \quad z \in \mathbb{C}_{+} .
$$


Then there is a sequence $f_{k} \in H^{\infty}\left(\mathbb{C}_{+}\right) \cap H^{1}\left(\mathbb{C}_{+}\right) \cap A^{1}(m)$ such that

$$
\left\|f_{k} e_{\lambda} S-\varphi_{N, n, \lambda_{1}, \delta}\right\|_{A^{1}(m)} \rightarrow 0 \quad \text { as } k \rightarrow+\infty
$$

for all $m-n>-1, N>0, \lambda_{1}>\lambda \geq 0$.

Proof. By Lemma 4, we have

$$
\varphi_{N, n, \lambda_{1}, \delta}(z)=S^{\delta}(z) e^{i \lambda_{1} z} \psi_{n, \lambda_{1}, \delta}(z) \frac{N^{2}}{(i N+z)^{2}}, \quad z \in \mathbb{C}_{+} .
$$

Consequently, for an arbitrary sequence $\left\{f_{k}\right\}_{k=1}^{\infty}$ in $H\left(\mathbb{C}_{+}\right)$, we can write

$$
f_{k}(z) e_{\lambda}(z) S(z)-\varphi_{N, n, \lambda_{1}, \delta}(z)=e_{\lambda}(z) S(z)\left(f_{k}(z)-\tilde{\varphi}_{N, n, \lambda_{1}, \lambda, \delta}(z)\right),
$$

where $\tilde{\varphi}_{N, n, \lambda_{1}, \lambda, \delta}(z)=\frac{\varphi_{N, n, \lambda_{1}, \delta}(z)}{e_{\lambda}(z) S(z)}, z \in \mathbb{C}_{+}$. Obviously, $e_{\lambda} S \in H^{\infty}\left(\mathbb{C}_{+}\right)$for all $\lambda \geq 0$. Since the set $H^{\infty}\left(\mathbb{C}_{+}\right) \cap H^{1}\left(\mathbb{C}_{+}\right) \cap A^{1}(m)$ is dense in $A^{1}(m)$ for all $m>-1$, it suffices to show that $\tilde{\varphi}_{N, n, \lambda_{1}, \lambda, \delta} \in A^{1}(m)$ if $m-n>-1$. For this, we observe that

$$
\tilde{\varphi}_{N, n, \lambda_{1}, \lambda, \delta}(z)=S^{\delta-1}(z) e^{i\left(\lambda_{1}-\lambda\right) z} \psi_{n, \delta, \lambda_{1}}(z) \frac{N^{2}}{(z+i N)^{2}}, \quad z \in \mathbb{C}_{+} .
$$

We have

$$
\begin{aligned}
\left\|\tilde{\varphi}_{N, n, \lambda_{1}, \lambda, \delta}\right\|_{A^{1}(m)}= & \int_{0}^{1} \int_{-\infty}^{+\infty}\left|\tilde{\varphi}_{N, n, \lambda_{1}, \lambda, \delta}(x+i y)\right| y^{m} d x d y \\
& +\int_{1}^{+\infty} \int_{-\infty}^{+\infty}\left|\tilde{\varphi}_{N, n, \lambda_{1}, \lambda, \delta}(x+i y)\right| y^{m} d x d y \\
= & I_{1}+I_{2}
\end{aligned}
$$

We estimate these integrals separately. First,

$$
I_{1} \leq \int_{0}^{1} y^{m} \int_{-\infty}^{+\infty} e^{-\left(\lambda_{1}-\lambda\right) y}|S(x+i y)|^{\delta-1}\left|\psi_{n, \delta, \lambda_{1}}(x+i y)\right| \frac{N^{2}}{\left(x^{2}+(y+N)^{2}\right)} d x d y .
$$

Using Lemma 4, we obtain

$$
I_{1} \leq c\left(n, \lambda_{1}\right) \sum_{p=1}^{n} \int_{0}^{1} \int_{-\infty}^{+\infty} y^{m-n} e^{-\left(\lambda_{1}-\lambda\right) y}|S(x+i y)|^{\delta-1}(U(x+i y) \delta y)^{p} \frac{N^{2}}{x^{2}+(y+N)^{2}} d y .
$$

We take into account the identity

$$
|S(x+i y)|=\exp \left\{-\frac{1}{\pi} \int_{-\infty}^{+\infty} \frac{y d \nu(t)}{(x-t)^{2}+y^{2}}\right\}
$$

and put

$$
\begin{aligned}
I_{1, p}=\int_{0}^{1} \int_{-\infty}^{+\infty} y^{m-n} e^{-\left(\lambda_{1}-\lambda\right) y} \exp \left\{-(\delta-1) \int_{-\infty}^{+\infty} \frac{y d \nu(t)}{y^{2}+(x-t)^{2}}\right\} \\
\times\left(\int_{-\infty}^{+\infty} \frac{y}{y^{2}+(x-t)^{2}} d \nu(t)\right)^{p} \frac{\delta^{p} N^{2} d x d y}{x^{2}+(y+N)^{2}}, \quad 1 \leq p \leq n .
\end{aligned}
$$

Obviously,

$$
\begin{aligned}
I_{1, p} \leq \int_{0}^{1} \int_{-\infty}^{+\infty} y^{m-n} \exp \{-(\delta-1) & \left.\int_{-\infty}^{+\infty} \frac{y}{y^{2}+(x-t)^{2}} d \nu(t)\right\} \\
& \times\left(\int_{-\infty}^{+\infty} \frac{y}{y^{2}+(x-t)^{2}} d \nu(t)\right)^{p} \frac{\delta^{p} N^{2} d x d y}{x^{2}+(y+N)^{2}}
\end{aligned}
$$


Observe that

$$
e^{-A(\delta-1)} A^{p} \leq\left(\frac{p}{\delta-1}\right)^{p} e^{-p}=c(p)
$$

for all $A>0$ and $\delta>1$. Therefore,

$$
\begin{array}{r}
I_{1, p} \leq c(p) \int_{0}^{1} y^{m-n} \int_{-\infty}^{+\infty} \frac{\delta^{p} N^{2} d x}{x^{2}+(y+N)^{2}} d y=c(p) \delta^{p} N^{2} \int_{0}^{1} \frac{y^{m-n}}{y+N} d y=c_{1}(p, \delta, N), \\
p=1,2, \ldots, n .
\end{array}
$$

So, $I_{1} \leq C_{2}(\delta, N)$ for $m-n>-1$.

To estimate $I_{2}$, we use Lemma 4 once again:

$$
\begin{aligned}
I_{2} \leq c\left(n, \lambda_{1}\right) \sum_{p=1}^{n} \int_{1}^{+\infty} \int_{-\infty}^{+\infty} & \frac{y^{m} N^{2}}{x^{2}+(N+y)^{2}}\left[\left(\delta \int_{-\infty}^{+\infty} \frac{y d \nu(t)}{(x-t)^{2}+y^{2}}\right)^{p}+1\right] \\
& \times \exp \left\{-\left(\lambda_{1}-\lambda\right) y-(\delta-1) \int_{-\infty}^{+\infty} \frac{y d \nu(t)}{(x-t)^{2}+y^{2}}\right\} d x d y .
\end{aligned}
$$

Recalling (19), we arrive at the inequality

$$
\begin{aligned}
I_{2} & \leq c_{1}\left(n, \lambda_{1}, \delta\right) \sum_{p=1}^{n} \int_{1}^{+\infty} y^{m} e^{-\left(\lambda_{1}-\lambda\right) y} \int_{-\infty}^{+\infty} \frac{N^{2}}{x^{2}+(N+y)^{2}} d x d y \\
& \leq c_{2}\left(n, \lambda_{1}, \delta\right) \int_{1}^{+\infty} \frac{y^{m} e^{-\left(\lambda_{1}-\lambda\right) y} N^{2}}{y+N} d y \leq c_{3}\left(n, \lambda_{1}, \lambda, \delta\right) N
\end{aligned}
$$

(we have used the condition $\lambda_{1}>\lambda \geq 0$ ). Estimates (20) and (21) complete the proof.

Lemma 6. Suppose that the Fourier transform of a function $f \in N\left(\mathbb{C}_{+}\right) \cap L^{1}(\mathbb{R})$ satisfies estimate (5) with a rapidly growing $\lambda$ on $(0,+\infty)$. Put

$$
f_{ \pm}(z)=\frac{1}{2 \pi i} \int_{-\infty}^{+\infty} \frac{f(t)}{t-z} d t
$$

for $z \in \mathbb{C}_{ \pm}$(respectively). Then $f_{-} \in C_{A}^{\infty}\left(\mathbb{C}_{-}\right)$, and $f_{+} \varphi_{N} \in H^{1}\left(\mathbb{C}_{+}\right)$for all $N>0$, where $\varphi_{N}(z)=\frac{N^{2}}{(i N+z)^{2}}$, as before.

Proof. By using the relation

$$
\widehat{f}(x)=\frac{1}{\sqrt{2 \pi}} \int_{-\infty}^{+\infty} f(t) e^{-i t x} d t=\frac{1}{\sqrt{2 \pi}} \int_{-\infty}^{0} f(t) e^{-i t x} d t+\frac{1}{\sqrt{2 \pi}} \int_{0}^{+\infty} f(t) e^{-i t x} d t
$$

and the Parseval identity (see [10, p. 11]), it is easy to show that the function $f(z)$ admits the representation

$$
f_{-}(z)=-\frac{1}{\sqrt{2 \pi}} \int_{-\infty}^{0} \widehat{f}(t) e^{i t z} d t
$$

Therefore, $f_{-} \in \mathbb{C}_{A}^{\infty}\left(\mathbb{C}_{-}\right)$.

The Sokhotskil theorem says that

$$
f(x)=f_{+}(x)-f_{-}(x)
$$

a.e. on $\mathbb{R}$; since $f_{-} \in L^{\infty}(\mathbb{R})$, we have $\left|f_{+}(x)\right| \leq|f(x)|+\left|f_{-}(x)\right|$, i.e.,

$$
\frac{f_{+}(x) N^{2}}{N^{2}+x^{2}} \in L^{1}(\mathbb{R})
$$

Applying the Smirnov theorem (see [11, p. 72]), we see that $\frac{f_{+}(z) N^{2}}{(i N+z)^{2}} \in H^{1}\left(\mathbb{C}_{+}\right)$. 
Lemma 7. Let $f_{+}$and $f_{-}$be as in Lemma 6 , and let

$$
f(z)=\frac{\psi_{1}(z)}{\psi_{2}(z)}, \quad z \in \mathbb{C}_{+}, \quad \psi_{j} \in H^{\infty}\left(\mathbb{C}_{+}\right), \quad j=1,2 .
$$

If $\psi_{2}(z)=e^{i a z} S(z) h(z)$, where $a \geq 0, S$ is a singular inner function, and $h$ is an outer function on $\mathbb{C}_{+}$, then

$$
\int_{-\infty}^{+\infty} f_{-}(x) e^{i a x} S(x) g(x) d x=0 \quad \text { for all } g \in H^{1}\left(\mathbb{C}_{+}\right) .
$$

Proof. We multiply (22) by $\varphi_{N} \psi_{2}$, obtaining

$$
f_{+}(x) \varphi_{N}(x) \psi_{2}(x)-f_{-}(x) \varphi_{N}(x) \psi_{2}(x)=\psi_{1}(x) \varphi_{N}(x) .
$$

By Lemma 6 , we have $f_{+} \varphi_{N} \in H^{1}\left(\mathbb{C}_{+}\right)$, and $\psi_{2} \in H^{\infty}\left(\mathbb{C}_{+}\right)$by the hypothesis. Since $f_{-} \varphi_{N} \psi_{2}, \psi_{1} \varphi_{N} \in L^{\infty}(\mathbb{R})$, the Smirnov theorem shows that $f_{+} \varphi_{N} \psi_{2} \in H^{\infty}\left(\mathbb{C}_{+}\right)$. Therefore,

$$
f_{-}(x) \varphi_{N}(x) \psi_{2}(x) g(x)=g(x) \varphi_{N}(x) \psi_{2}(x) f_{+}(x)-\psi_{1}(x) \varphi_{N}(x) g(x)
$$

for any $g \in H^{1}\left(\mathbb{C}_{+}\right)$. This implies that

$$
g \varphi_{N} \psi_{2} f_{+} \in H^{1}\left(\mathbb{C}_{+}\right),
$$

whence

$$
\int_{-\infty}^{+\infty} f_{-}(x) \varphi_{N}(x) \psi_{2}(x) g(x) d x=0
$$

i.e.,

$$
\int_{-\infty}^{+\infty} f_{-}(x) \varphi_{N}(x) e^{i a x} S(x) h(x) g(x) d x=0 .
$$

Since $\psi_{2} \in H^{\infty}\left(\mathbb{C}_{+}\right)$by the assumptions of the lemma, we have $h \in H^{\infty}\left(\mathbb{C}_{+}\right)$. By a well-known theorem (see [11, Theorem 7.4]), there exists a sequence of outer functions $f_{n} \in H^{\infty}\left(\mathbb{C}_{+}\right)$such that $\left\|f_{n} h\right\|_{\infty} \leq 1$ and $f_{n}(x) h(x) \rightarrow 1$ a.e. on $\mathbb{R}$. Replacing $g(x)$ in (23) with functions of the form $f_{n} g$ and passing to the limit as $n \rightarrow \infty$, we obtain

$$
\int_{-\infty}^{+\infty} f_{-}(x) e^{i a x} S(x) \varphi_{N}(x) g(x) d x=0, \quad g \in H^{1}\left(\mathbb{C}_{+}\right) .
$$

Now, to complete the proof, it suffices to let $N$ tend to $+\infty$.

Lemma 8. Let $\lambda(x)>0$ be a monotone increasing function on $(0,+\infty)$ such that $\int_{1}^{+\infty} \frac{\ln \lambda(x)}{x^{3 / 2}} d x<+\infty$. Then there exists an entire function $G(z)$ of order $\frac{1}{2}$ and of normal type, with $G(0)=G^{\prime}(0)=0, G(x) \neq 0$ for $x<0$, and such that

$$
|G(x)| \leq \frac{1}{\lambda(-x)}, \quad x \in \mathbb{R}_{-} .
$$

Proof. There is no loss of generality in assuming that $\lambda(x)>\exp \left(x^{\beta}\right)$ for $x>x_{0}$, where $0<\beta<1 / 2$. Indeed, otherwise we can take $\tilde{\lambda}(x)=\lambda(x) \exp \left(x^{\beta}\right), x \geq 0$, instead of $\lambda(x)$. Under the conditions of the lemma, there exists an even entire function $\tilde{F}(z)$ such that $|\tilde{F}(z)| \leq \exp (c|z|), z \in \mathbb{C}$, and $|\tilde{F}(x)| \leq \frac{1}{\left(\lambda\left(x^{2}\right)\right)^{2}}, \tilde{F}(x) \neq 0, x \in(-\infty,+\infty)$ (see [4]).

Put $F(z)=z^{4} \tilde{F}(z)$. Clearly,

$$
|F(z)| \leq \exp \left(c_{1}|z|\right), \quad z \in \mathbb{C}, \quad|F(x)| \leq \frac{c_{2}}{\lambda\left(x^{2}\right)}, \quad F(x) \neq 0, \quad x \in \mathbb{R},
$$

for some $c_{2}$. Now we define $G$ as follows: $G(z)=\frac{F(\sqrt{-z})}{c_{2}}$. Then, obviously, $|G(x)|=$ $|F(\sqrt{-x})| \leq \frac{1}{\lambda(-x)}$. Thus, the function $G$ is as required. 
The next lemma was established in [12].

Lemma 9. Suppose $P$ is a monotone increasing, continuous, and positive function on $(0,+\infty)$. Assume that $\int_{1}^{+\infty} \frac{P(r)}{r^{2}} d r<+\infty$ and that the function $\frac{P(r)}{r}$ is monotone decreasing on $\mathbb{R}_{+}$. Then there exists an entire function $G$ of exponential type such that

$$
|G(z)| \leq \exp (-P(|z|)), \quad G(z) \neq 0, \quad z \in \mathbb{C}_{+} \cup \mathbb{R} .
$$

Lemma 10. Let $G$ be the function constructed in Lemma $8, G(z)=\sum_{n=2}^{+\infty}(-1)^{n} A_{2 n} z^{n}$, where the $A_{2 n}$ are the coefficients of the expansion of the function $F$ occurring in the proof of Lemma 8, and

$$
H(z)=\sum_{n=2}^{+\infty}(-1)^{n} A_{2 n} n ! z^{n} .
$$

Then $H$ is an entire function of exponential type, and

$$
\int_{|t|<1} \frac{|H(i t)|}{t^{2}} d t<+\infty
$$

The proof follows readily from Lemma 8, the Stirling formula, and the relationship between the growth of an entire function and the rate of decay of the coefficients in its expansion.

\section{§2. Proof of the main theorems}

First, we prove Theorem 1 . It is easily seen that it suffices to check that the function $f_{-}(z)$ constructed in Lemma 6 is identically zero. We put

$$
\Phi(g)=\lim _{y \rightarrow 0+} \int_{-\infty}^{+\infty} f_{-}(x-i y) g(x+i y) d x .
$$

By Lemmas 2 and $6, \Phi$ is a continuous linear functional on each space $A^{1}(m)$ for all $m \in \mathbb{Z}_{+}$. By Lemma 7 ,

$$
\int_{-\infty}^{+\infty} e^{i \lambda x} e^{i a x} S(x) g(x) f_{-}(x) d x=0
$$

for all $\lambda \geq 0$ and all $g \in H^{1}\left(\mathbb{C}_{+}\right) \cap A^{1}(m)$. Recalling Lemmas 2 and 5, we obtain

$$
\lim _{y \rightarrow 0+} \int_{-\infty}^{+\infty} f_{-}(x-i y)\left(e^{i \lambda_{1}(x+i y)} S^{\delta}(x+i y) \frac{N^{2}}{(i N+x+i y)^{2}} d x\right)^{(n)}=0,
$$

where $n=0,1, \ldots, \delta>1$, and $\lambda_{1}>a \geq 0$.

Put $\varphi_{N, \delta, \lambda}(z) \stackrel{\text { def }}{=} e^{i \lambda z} S^{\delta}(z) \frac{N^{2}}{(i N+z)^{2}}, z \in \mathbb{C}_{+}$. Let $\widehat{\varphi}_{N, \delta, \lambda}$ denote the Fourier transform of $\varphi_{N, \delta, \lambda}$. By the Parseval identity

$$
\int_{-\infty}^{+\infty} F(x) G(x) d x=\int_{-\infty}^{+\infty} \widehat{F}(x) \widehat{G}(-x) d x, \quad F, G \in L^{2}
$$

(see [10]), we have

$$
\int_{-\infty}^{+\infty} f_{-}(x-i y) \varphi_{N, \delta, \lambda}^{(n)}(x+i y) d x=i^{n} \int_{-\infty}^{+\infty} \widehat{f}_{-, y}(x) \widehat{\varphi}_{N, \delta, \lambda, y}(-x) x^{n} d x
$$


where $\widehat{f}_{-, y}(x)$ is the Fourier transform of $f_{-}(x-i y)$ and $\widehat{\varphi}_{N, \delta, \lambda, y}$ is the Fourier transform of $\varphi_{N, \delta, \lambda}(x+i y)$. The Paley-Wiener theorem (see [1, p. 187]) yields

$$
\begin{aligned}
f_{-}(x-i y) & =\frac{1}{\sqrt{2 \pi}} \int_{-\infty}^{0} \widehat{f}_{-, y}(t) e^{i t z} d t, \quad z=x+i y, \quad y>0, \\
\varphi_{N, \delta, \lambda}(x+i y) & =\frac{1}{\sqrt{2 \pi}} \int_{0}^{+\infty} \widehat{\varphi}_{N, \delta, \lambda}(t) e^{i t z} d t, \quad \operatorname{Im} z>0 .
\end{aligned}
$$

Therefore,

$$
\begin{aligned}
\widehat{\varphi}_{N, \delta, \lambda}(u) & =\frac{1}{\sqrt{2 \pi}} \int_{-\infty}^{+\infty} \varphi_{N, \delta, \lambda}(t) e^{-i t u} d t \\
& =\frac{1}{\sqrt{2 \pi}} \int_{-\infty}^{+\infty} S^{\delta}(t) \frac{N^{2}}{(i N+t)^{2}} e^{-i t(u-\lambda)} d t=\widehat{\varphi}_{N, \delta}(u-\lambda),
\end{aligned}
$$

where $\varphi_{N, \delta}(x)=\varphi_{N, \delta, 0}(x)=\frac{S^{\delta}(x) N^{2}}{(i N+x)^{2}}$.

Using formula (28) once again, as a result we obtain

$$
\int_{-\infty}^{+\infty} f_{-}(x-i y) \varphi_{N, \delta, \lambda}^{(n)}(x+i y) d x=i^{n} \int_{-\infty}^{0} \widehat{f}_{-}(t) e^{2 t y} \widehat{\varphi}_{N, \delta}(-t-\lambda) t^{n} d t, \quad y>0 .
$$

Now, we apply (24) and arrive at the identities

$$
\lim _{y \rightarrow 0+} \int_{-\infty}^{+\infty} \widehat{f}_{-}(t) \widehat{\varphi}_{N, \delta}(-t-\lambda) e^{2 t y} t^{n} d t=0, \quad n=0,1,2, \ldots, \quad \lambda>a \geq 0, \quad \delta>1 .
$$

The properties of the functions $\widehat{f}_{-}$and $\widehat{\varphi}_{N, \delta}$ show that

$$
\int_{-\infty}^{0} \widehat{f}_{-}(t) \widehat{\varphi}_{N, \delta}(-t-\lambda) t^{n} d t=0, \quad n=0,1, \ldots, \quad \lambda>a \geq 0, \quad \delta>1 .
$$

Consider the function

$$
G(z)=\int_{-\infty}^{0} \frac{\widehat{f}_{-}(t) \widehat{\varphi}_{N, \delta}(-t-\lambda)}{t+z^{2}} d t, \quad \operatorname{Im} z \neq 0 .
$$

We use a method well known in the weighted approximation theory (see [13]). The function $G$ is holomorphic on the upper half-plane. Since

$$
\frac{1}{t+z^{2}}=\sum_{k=0}^{n} \frac{(-1)^{k} t^{k}}{z^{2 k+2}}+\frac{(-1)^{n+1} t^{n+1}}{z^{2(n+1)}\left(t+z^{2}\right)}
$$

we have

$$
G(z)=\frac{(-1)^{n+1}}{z^{2(n+1)}} \int_{-\infty}^{0} \frac{\widehat{f}_{-}(t) \widehat{\varphi}_{N, \delta}(-t-\lambda)}{t+z^{2}} t^{n+1} d t .
$$


In the next calculation, we apply the Cauchy-Bunyakovski inequality, the Parseval identity, and the relation $\widehat{f}_{-}(t)=-\widehat{f}(t)$ for $t \in \mathbb{R}_{-}$. We have

$$
\begin{aligned}
|G(z)| & \leq \frac{1}{|z|^{2(n+1)}}\left(\int_{-\infty}^{0} \frac{|\widehat{f}(t)|^{2}|t|^{2(n+1)}}{\left|z^{2}+t\right|^{2}} d t\right)^{1 / 2}\left(\int_{-\infty}^{0}\left|\widehat{\varphi}_{N, \delta}(-t-\lambda)\right|^{2} d t\right)^{1 / 2} \\
& \leq \frac{1}{|z|^{2(n+1)}}\left(\int_{-\infty}^{0} \frac{|t|^{2(n+1)}}{\lambda^{2}(-t)\left|z^{2}+t\right|^{2}} d t\right)^{1 / 2}\left(\int_{-\infty}^{+\infty}\left|\widehat{\varphi}_{N, \delta}(t)\right|^{2} d t\right)^{1 / 2} \\
& =\frac{2}{|z|^{2(n+1)}}\left(\int_{0}^{+\infty}\left(\frac{t^{n+1}}{\lambda(t)}\right)^{2} \frac{d t}{\left((x+\sqrt{t})^{2}+y^{2}\right)\left((x-\sqrt{t})^{2}+y^{2}\right)}\right)^{1 / 2} \\
& \times\left(\int_{0}^{+\infty} \frac{N^{4}}{\left(t^{2}+N^{2}\right)^{2}} d t\right)^{1 / 2} \\
& \leq \frac{C(N)}{|z|^{2(n+1)}}\left(\int_{0}^{+\infty} \frac{t^{2(n+1)} d t}{\lambda(t)^{2}\left(y^{2}+t\right)}\right)^{1 / 2} \leq \frac{C_{1}(N)}{|z|^{2(n+1)}} \sup _{t \in \mathbb{R}_{+}}\left(\frac{t^{n+2}}{\lambda(t)}\right) \\
& =C_{1}(N) \frac{m_{n+2}}{|z|^{2(n+1)}}, \quad \operatorname{Im} z \geq 1 .
\end{aligned}
$$

Recall that the numbers $m_{n}, n \in \mathbb{N}$, are defined by formulas (3). Thus,

$$
\frac{|G(z)|}{|z|^{2}} \leq C_{1}(N) \frac{m_{n+2}}{|z|^{2(n+2)}}, \quad n=1,2, \ldots, \quad \operatorname{Im} z \geq 1 .
$$

Taking the infimum over $n$ and applying the uniqueness theorem for the class $N\left(\mathbb{C}_{+}\right)$ (see [4]), we see that $G(z)=0$ for $\operatorname{Im} z>0$. In the same way we can check that $G(z)=0$ for $\operatorname{Im} z<0$. Therefore,

$$
\widehat{f}_{-}(t) \widehat{\varphi}_{N, \delta}(-t-\lambda)=0, \quad t \in \mathbb{R}, \quad \lambda>a \geq 0 .
$$

Now, suppose there exists $x_{0} \in(-\infty, 0)$ with $\widehat{f}\left(x_{0}\right) \neq 0$. Then the above formula implies that

$$
\widehat{\varphi}_{N, \delta}\left(-x_{0}-\lambda\right)=0 \text { for all } \lambda>a \geq 0 .
$$

Therefore, $\widehat{\varphi}_{N, \delta}(u)=0$ for $u \leq\left|x_{0}\right|-a$. Consequently, if $\inf _{x \in \operatorname{supp}} x=-\infty$, then $\widehat{\varphi}_{N, \delta}(u)=0$ for all $u \in \mathbb{R}$, so that $\varphi_{N, \delta}=0$ a.e. on $\mathbb{R}$, which is impossible. Thus, there exists $-\infty<\alpha \leq 0$ such that $\widehat{f}_{-}(x)=0$ for all $-\infty<x<\alpha$. Then

$$
f_{-}(x)=\frac{1}{\sqrt{2 \pi}} \int_{\alpha}^{0} \widehat{f}_{-}(t) e^{i t x} d t
$$

i.e., $f_{-}$is an entire function of exponential type, $\sup _{z \in \mathbb{C}_{-}}\left|f_{-}(z)\right|=M<+\infty$.

We shall prove that

$$
\limsup _{y \rightarrow+\infty} \frac{\ln \left|f_{-}(i y)\right|}{y} \leq 0
$$

(by the Phragmèn-Lindelöf theorem, (29) implies that $f_{-}(z)=0$ for all $z \in \mathbb{C}$ ). We use (22). Since $f \in N\left(\mathbb{C}_{+}\right)$, by the uniqueness theorem we have $f(z)=f_{+}(z)-f_{-}(z)$, $\operatorname{Im} z>0$, i.e.,

$$
\ln \left|f_{-}(i y)\right| \leq \ln ^{+}\left|f_{-}(i y)\right| \leq \ln 2+\ln ^{+}|f(i y)|+\ln ^{+}\left|f_{+}(i y)\right| \leq \ln 2+\ln ^{+}|f(i y)|+\frac{c}{y} .
$$

By the assumptions of the theorem, this implies (29), so that $f_{-}(z)=0, z \in \mathbb{C}$. Consequently,

$$
f_{+}(z)=\frac{\psi_{1}(z)}{e^{i a z} S(z) h(z)}, \quad \operatorname{Im} z>0 .
$$


Since $f_{+}$belongs to the Smirnov class on the upper half-plane, we see that $a=0$, $S(z)=1, z \in \mathbb{C}_{+}$, and $f_{+} \in L^{1}(\mathbb{R})$. Applying Smirnov's theorem once again, we conclude that $f_{+} \in H^{1}\left(\mathbb{C}_{+}\right)$. This proves the first part of the theorem.

We show the converse statement. Let $G(z)$ be the function constructed in Lemma 8 and satisfying (25), and let $H(z)$ be the entire function constructed in Lemma 10. Put

$$
f(z)=\frac{1}{\sqrt{2 \pi}} \int_{-\infty}^{0} G(t) e^{i t z} d t, \quad \operatorname{Im} z<0 .
$$

Expanding the entire function $G(z)$ in a power series and using (27), we see that

$$
f(z)=H\left(\frac{1}{-i z}\right), \quad z \in \mathbb{C}, \quad z \neq 0 .
$$

We show that $f(z)$ satisfies all the requirements of the theorem. Obviously,

$$
\widehat{f}(x)=\frac{1}{\sqrt{2 \pi}} \int_{-\infty}^{+\infty} f(t) e^{-i t x} d t= \begin{cases}G(x) & \text { if } x<0 \\ 0 & \text { if } x \geq 0\end{cases}
$$

Therefore, $|\widehat{f}(x)| \leq \frac{1}{\lambda(|x|)}, x \in(-\infty,+\infty)$. Moreover, Lemma 8 shows that $\widehat{f}(x) \neq 0$, $x \in \mathbb{R}_{-}$. We check that $f \in L^{1}(\mathbb{R})$.

Identity (30) implies the inequality

$$
|f(x)| \leq \frac{1}{\sqrt{2 \pi}} \int_{-\infty}^{0}|G(x)| d x \leq \frac{1}{\sqrt{2 \pi}} \int_{-\infty}^{0} \frac{d x}{\lambda(-x)}<+\infty .
$$

Thus, we need to estimate $\int_{|x| \geq 1}|f(x)| d x$. By (31), we have

$$
\int_{|x| \geq 1}|f(x)| d x=\int_{|x| \geq 1}\left|H\left(-\frac{1}{i x}\right)\right| d x=\int_{|x| \leq 1} \frac{|H(i x)|}{x^{2}} d x<+\infty .
$$

Since $H(z)$ is an entire function with $H(0)=H^{\prime}(0)=0$, we have $f \in L^{1}(\mathbb{R})$.

It remains to prove that $f$ is a function of bounded type in $\mathbb{C}_{+}$. With the help of (30), it is easy to show that $\sup _{z \in \mathbb{C}_{-}}|f(z)|<+\infty$.

Using (31), we observe that $\sup _{x \in \mathbb{R}_{-}}|H(x)|<+\infty$, and the Phragmèn-Lindelöf theorem yields $\sup _{\operatorname{Re} z<0}|H(z)|<+\infty$. We put $\Phi(z)=H(z) e^{-B z}, B>0, z \in \mathbb{C}$. Then $\Phi$ is an entire function of exponential type. We estimate it in the right half-plane. We have

$$
\sup _{y \in \mathbb{R}}|\Phi(i y)|=\sup _{y \in \mathbb{R}}|H(i y)|<+\infty \text {. }
$$

At the same time,

$$
|\Phi(x)|=|H(x)| e^{-B x}, \quad x>0 .
$$

Since $H(z)$ is an entire function of exponential type, we have $|H(z)| \leq c e^{\sigma|z|}, z \in \mathbb{C}_{+}$. Choosing $B>\sigma$ and using the Phragmèn-Lindelöf theorem, we obtain

$$
|H(z)| \leq M\left|e^{B z}\right|, \quad \operatorname{Re} z>0 .
$$

By (31), it follows that

$$
|f(z)|=\left|H\left(\frac{i}{z}\right)\right| \leq M_{1}\left|e^{\frac{i B}{z}}\right|, \quad \operatorname{Im} z>0 .
$$

Therefore, $|f(x+i y)| \leq M_{1} e^{\frac{B y}{x^{2}+y^{2}}}, y>0$. Consequently,

$$
\sup _{0<y<+\infty} \int_{-\infty}^{+\infty} \frac{\ln ^{+}|f(x+i y)|}{1+x^{2}} d x<+\infty ;
$$

i.e., $f$ is a function of bounded type. 
Remark 5. The above proof shows that the first part of the theorem remains valid if we replace the condition $f \in N\left(\mathbb{C}_{+}\right) \cap L^{1}(\mathbb{R})$ by the condition $f \in N\left(\mathbb{C}_{+}\right) \cap L^{p_{0}}(\mathbb{R})$ for some $1 \leq p_{0} \leq 2$.

Proof of Theorem 3. Let $G$ be the entire function of exponential type constructed in Lemma 9. Assume that $P(r) \geq r^{\beta}, 0<\beta<1$. The construction implies that $G \in$ $H^{\infty}\left(\mathbb{C}_{+}\right), G \neq 0$ for $z \in \mathbb{C}_{+}$, and $G$ is an outer function in the upper half-plane. We use estimate (26), put $F(z)=G(z) \cdot f(z), z \in \mathbb{C}_{+}$, and observe that $F \in N\left(\mathbb{C}_{+}\right) \cap L^{1}(\mathbb{R})$. To estimate $F^{(n)}(x), x \in \mathbb{R}$, we apply the Leibniz formula:

$$
\left|F^{(n)}(x)\right| \leq \sum_{k=0}^{n}\left(\begin{array}{l}
n \\
k
\end{array}\right)\left|f^{(n-k)}(x)\right| \cdot\left|G^{(k)}(x)\right| \leq A_{f}^{n} M_{n} \sum_{k=0}^{n}\left(\begin{array}{l}
n \\
k
\end{array}\right)\left|G^{(k)}(x)\right|, \quad x \in \mathbb{R} .
$$

Consequently,

$$
\int_{-\infty}^{+\infty}\left|F^{(n)}(x)\right| d x \leq A_{f}^{n} M_{n} \sum_{k=0}^{n}\left(\begin{array}{l}
n \\
k
\end{array}\right) \int_{-\infty}^{+\infty}\left|G^{(k)}(x)\right| d x .
$$

Since $G$ is an entire function of exponential type, a Bernstein type inequality (see [14]) shows that

$$
\int_{-\infty}^{+\infty}\left|G^{(k)}(x)\right| d x \leq \sigma^{k} \int_{-\infty}^{+\infty}|G(x)| d x \leq 2 \sigma^{k} \int_{0}^{+\infty} e^{-P(r)} d r=C \sigma^{k} .
$$

Now, using (33), we obtain

$$
\int_{-\infty}^{+\infty}\left|F^{(n)}(x)\right| d x \leq A_{f}^{n} M_{n} C \sum_{k=0}^{+\infty}\left(\begin{array}{l}
n \\
k
\end{array}\right) \sigma^{k}
$$

i.e.,

$$
\int_{-\infty}^{+\infty}\left|F^{(n)}(x)\right| d x \leq A_{f}^{n} M_{n} C(1+\sigma)^{n}=A_{1, f}^{n} M_{n}, \quad n=0,1,2, \ldots .
$$

Consider the Fourier transform of $F$ :

$$
\widehat{F}(x)=\frac{1}{\sqrt{2 \pi}} \int_{-\infty}^{+\infty} F(t) e^{-i t x} d t .
$$

Integration by parts yields

$$
\widehat{F}(x)=\frac{(-1)^{n}}{\sqrt{2 \pi}(i x)^{n}} \int_{-\infty}^{+\infty} F^{(n)}(t) e^{-i t x} d t
$$

whence

$$
|\widehat{F}(x)| \leq \frac{A_{1}^{n} M_{n}}{|x|^{n}}, \quad n=1,2, \ldots, \quad x \in \mathbb{R} .
$$

Choosing $n$ appropriately, we see that

$$
|\widehat{F}(x)| \leq \frac{1}{T\left(\frac{|x|}{A_{1}}\right)}, \quad x \in \mathbb{R} .
$$

Also, we have

$$
\ln |F(i y)|=\ln |f(i y)|+\ln |G(i y)| .
$$

By (9) and (26), this implies

$$
\limsup _{y \rightarrow+\infty} \frac{\ln |F(i y)|}{y} \leq 0 .
$$

Thus, all the assumptions of Theorem 1 are fulfilled. By that theorem, $\widehat{F}(x)=0$ for $x \in$ $\mathbb{R}_{-}$. Moreover, $F \in H^{1}\left(\mathbb{C}_{+}\right)$, and since $G$ is outer and $f \in L^{1}(\mathbb{R})$, we have $f \in H^{1}\left(\mathbb{C}_{+}\right)$ 
by the Smirnov theorem. Therefore, in the upper half-plane, $f$ can be represented by a Poisson integral:

$$
f(z)=\frac{1}{2 \pi i} \int_{-\infty}^{+\infty} f(t)\left(\frac{1}{t-z}-\frac{1}{t-\bar{z}}\right) d t, \quad z \in \mathbb{C}_{+} .
$$

Consequently,

$$
f^{(n)}(z)=\frac{n !}{2 \pi i} \int_{-\infty}^{+\infty} \frac{f(t)}{(t-z)^{n+1}} d t
$$

Integrating by parts, we obtain

$$
f^{(n)}(x+i y)=\frac{1}{\pi} \int_{-\infty}^{+\infty} f^{(n)}(t) \frac{y}{(x-t)^{2}+y^{2}} d t
$$

whence

$$
\left|f^{(k)}(x+i y)\right| \leq A_{f}^{k} M_{k}, \quad k=0,1,2, \ldots .
$$

To prove the second part of the theorem, it remains to apply Lemmas 8 and 10 and to repeat the arguments in the proof of the second part of Theorem 1.

The next theorem can be proved in a similar way.

Theorem 4. Let $f \in N\left(\mathbb{C}_{+}\right)$be such that $f^{(n)} \in L^{1}(\mathbb{R}), n=0,1,2, \ldots$, and

$$
\int_{-\infty}^{+\infty}\left|f^{(n)}(t)\right| d t \leq A_{f}^{n} M_{n}, \quad n=0,1,2, \ldots
$$

Moreover, suppose that conditions (9) and (10) are fulfilled. Then $f \in H^{1}\left(\mathbb{C}_{+}\right)$and

$$
\int_{-\infty}^{+\infty}\left|f^{(n)}(x+i y)\right| d x \leq A_{f}^{n} M_{n}, \quad y>0, \quad n=0,1,2, \ldots .
$$

Theorems 1 and 3 imply the following refinement of a theorem by R. Salinas (see [15]).

Theorem 5. Let $g \in C^{\infty}\left(M_{n}\right)$. Suppose there exists $f \in N\left(\mathbb{C}_{+}\right)$such that

$$
\lim _{y \rightarrow 0} f(x+i y)=g(x)
$$

almost everywhere. Moreover, suppose that conditions (9) and (10) are fulfilled. If $g^{(n)}\left(x_{0}\right)=0$ for some $x_{0} \in \mathbb{R}, n=0,1, \ldots$, then $g(x)=0$ for all $x \in \mathbb{R}$.

\section{REFERENCES}

[1] K. Hoffman, Banach spaces of analytic functions, Prentice-Hall, Inc., Englewood Cliffs, NJ, 1962. MR $0133008(24: \mathrm{A} 2844)$

[2] P. Duren, Theory of $H^{p}$ spaces, Pure Appl. Math., vol. 38, Acad. Press, New York-London, 1970. MR $0268655(42: 3552)$

[3] F. A. Shamoyan, On Fourier transform of functions of bounded type, Scientific Conference Dedicated to the Academician I. G. Petrovskiı̆ Centenary: Thesis, Bryansk. Gos. Univ., Bryansk, 2001, pp. 2728. (Russian)

[4] S. Mandelbrojt, Séries adhérentes, régularisation des suites, applications, Gauthier-Villars, Paris, 1952. MR0051893 (14:542f)

[5] J.-P. Kahane and Y. Katznelson, Sur le comportement radial des fonctions analytiques, C. R. Acad. Sci. Paris Sér. A-B 272 (1971), A718-A719. MR0277721 (43:3454)

[6] F. A. Shamoyan, Characterization of the rate of decrease of the Fourier coefficients of functions of bounded type and classes of analytic functions with infinitely differentiable boundary values, Sibirsk. Mat. Zh. 36 (1995), no. 4, 943-953; English transl., Siberian Math. J. 36 (1995), no. 4, 816-826. MR $1367262(97 \mathrm{~b}: 30050)$

[7] L. Hörmander, An introduction to complex analysis in several variables, 3rd ed., North-Holland Math. Library, vol. 7, North-Holland Publ. Co., Amsterdam, 1990. MR1045639 (91a:32001) 
[8] M. M. Dzhrbashyan and A. È. Dzhrbashyan, Integral representation for certain classes of analytic functions in the half plane, Dokl. Akad. Nauk SSSR 285 (1985), no. 3, 547-550; English transl., Soviet Math. Dokl. 32 (1985), no. 3, 727-730. MR0821337 (87d:30042)

[9] N. Bourbaki, Éléments de mathématique. I: Les structures fondamentales de l'analyse. Livre IV. Fonctions d'une variable réelle (théorie élémentaire). Chapitres 1,2 et 3: Dérivées. Primitives et intégrales. Fonctions élémentaires, Actualités Sci. Indust., No. 1074, Hermann, Paris, 1958. MR0151354 (27:1340)

[10] R. Paley and N. Wiener, Fourier transforms in the complex domain, Amer. Math. Soc. Colloq. Publ., No. 19, Amer. Math. Soc., Providence, RI, 1987. MR1451142 (98a:01023)

[11] J. Garnett, Bounded analytic functions, Pure Appl. Math., vol. 96, Acad. Press, Inc., New YorkLondon, 1981. MR0628971 (83g:30037)

[12] M. M. Dzhrbashyan, On asymptotic approximation by entire functions in a half plane, Dokl. Akad. Nauk SSSR (N.S.) 111 (1956), no. 4, 749-752. (Russian) MR0086177 (19:138a)

[13] S. N. Mergelyan, Weighted approximations by polynomials, Uspekhi Mat. Nauk (N.S.) 11 (1956), no. 5, 107-152; English transl., Amer. Math. Soc. Transl. (2), vol. 10, Amer. Math. Soc., Providendce, RI, 1958, pp. 59-106. MR0083614 (18:734c)

[14] N. I. Akhiezer, Lectures in the theory of approximation, "Nauka", Moscow, 1965; English transl., Theory of approximation, Dover Publ., Inc., New York, 1992. MR0188672 (32:6108) MR1217081 (94b:01041)

[15] B. R.-Salinas, Functions with null moments, Rev. Acad. Ci. Madrid 49 (1955), 331-368. MR0080174 $(18: 204 \mathrm{e})$

Bryansk State University, 241050 Bryansk, Russia

E-mail address: shamoyan@tu-bryansk.ru

Received 5/JUL/2007

Translated by A. PLOTKIN 